\title{
The homotopy types of $U(n)$-gauge groups over lens spaces
}

\author{
Ingrid Membrillo-Solis ${ }^{1}$ (D) $\cdot$ Stephen Theriault ${ }^{1}$
}

Received: 9 August 2019 / Accepted: 12 October 2020 / Published online: 1 April 2021

(C) The Author(s) 2021

\section{Abstract}

We analyse the homotopy types of gauge groups for principal $U(n)$-bundles over lens spaces and two-dimensional Moore spaces.

Keywords Lens space - Gauge group · Homotopy type

Mathematics Subject Classification Primary 55P15 $\cdot$ 54C35 $\cdot$ Secondary 81T13

\section{Introduction}

Let $G$ be a simple, compact Lie group and $P \longrightarrow M$ be a principal $G$-bundle. The gauge group of this bundle is the group of $G$-equivariant automorphisms of $P$ that fix $M$. There has been considerable work recently in trying to understand the homotopy types of gauge groups that arise in physical or geometric contexts. Most work to date has concentrated on $M$ being a simply connected four-manifold when $G$ is simply connected or $M$ being an orientable surface when $G=U(n)$.

In this paper, we turn our attention to the case when $M$ is a 3-manifold. If $G$ is simply connected then $[M, B G] \cong 0$, implying that the only principal $G$-bundle is the trivial bundle, which has the trivial gauge group. We consider instead the more topologically intricate case when $G=U(n)$ and $M$ is a lens space, for then $[M, B U(n)] \not 0$.

Let $p$ and $q$ be coprime integers. The lens space $L(p, q)$ is the orbit space $S^{3} /(\mathbb{Z} / p \mathbb{Z})$, where the action of $\mathbb{Z} / p \mathbb{Z}$ on $S^{3}$ is given by $\left(z_{0}, z_{1}\right) \longrightarrow\left(e^{2 \pi i / p} z_{0}, e^{2 \pi i q / p} z_{1}\right)$. For $n \geq 1$ and $p \geq 2$, let $p: S^{n} \longrightarrow S^{n}$ be the map of degree $p$ and let $P^{n+1}(p)$ be its homotopy cofibre. The space $P^{n+1}(p)$ is the $(n+1)$-dimensional mod- $p$ Moore space. As a $C W$-complex, $L(p, q) \simeq P^{2}(p) \cup e^{3}$.

\footnotetext{
$凶 \quad$ Ingrid Membrillo-Solis

I.Membrillo-Solis@soton.ac.uk

Stephen Theriault

S.D.Theriault@soton.ac.uk

1 Mathematical Sciences, University of Southampton, Southampton SO17 1BJ, UK
} 
The analysis of gauge groups of principal $U(n)$-bundles over $L(p, q)$ is necessarily delicate for two reasons. First, the isomorphism classes of principal bundles is determined by $[L(p, q), B U(n)]$, and this set is determined by $\left[P^{2}(p), B U(n)\right]$ rather than $\left[S^{3}, B U(n)\right]$. This is in contrast to the case when $G$ is simply connected and $M$ is a simply connected four-manifold or when $G=U(n)$ and $M$ is an orientable surface; in both of those cases $[M, B G]$ is determined by the top cell of $M$ and this leads to certain homotopy fibrations being more easily compared. Second, typically localization techniques are used to work one prime at a time, allowing for easier progress. However, as $L(p, q)$ may not be nilpotent, localization techniques may be problematic, so we approach the problem without localization. The strategy and methods used should be applicable to other cases as well.

As will be shown, there are isomorphisms $[L(p, q), B U(n)] \cong\left[P^{2}(p), B U(n)\right] \cong$ $\mathbb{Z} / p \mathbb{Z}$. For $k \in \mathbb{Z} / p \mathbb{Z}$, let $\mathcal{G}_{k}(L(p, q))$ and $\mathcal{G}_{k}\left(P^{2}(p)\right)$, respectively, be gauge groups of the principal $U(n)$-bundles over $L(p, q)$ and $P^{2}(p)$ with first Chern class $k$. Our main results for $\mathcal{G}_{k}\left(P^{2}(p)\right)$ are stated in Proposition 3.4 and Corollary 3.6, and our main results for $\mathcal{G}_{k}(L(p, q))$ are stated in Proposition 4.3 and Corollary 4.6. For now, we point out two special cases that give classifications and are easier to state.

For integers $a, b$, let $(a, b)$ be their greatest common divisor.

Theorem 1.1 Let $p$ be a prime and consider the gauge groups of principal $U(p)$ bundles over $P^{2}(p)$ and $L(p, q)$. The following hold:

(a) $\mathcal{G}_{k}\left(P^{2}(p)\right) \simeq \mathcal{G}_{\ell}\left(P^{2}(p)\right)$ if and only if $(p, k)=(p, \ell)$;

(b) if $p \in\{3,5\}$ then $\mathcal{G}_{k}(L(p, q)) \simeq \mathcal{G}_{\ell}(L(p, q))$ if and only if $(p, k)=(p, \ell)$.

The authors would like to thank the referees for identifying an error in an earlier version of the paper, and for pointing out the work of Olum [6] and Lemma 2.4.

\section{Isomorphism classes of bundles and components of mapping spaces}

As a $C W$-complex $L(p, q) \simeq P^{2}(p) \cup e^{3}$, so there is a homotopy cofibration

$$
S^{2} \stackrel{f}{\longrightarrow} P^{2}(p) \stackrel{i}{\longrightarrow} L(p, q)
$$

where $f$ attaches the top cell to $L(p, q)$ and $i$ is the inclusion of the 2-skeleton. Let $\pi: P^{2}(p) \longrightarrow S^{2}$ be the pinch map to the top cell. Let $j$ be the composite of inclusions $j: S^{1} \longrightarrow P^{2}(p) \stackrel{i}{\longrightarrow} L(p, q)$. Then, there is a homotopy cofibration diagram

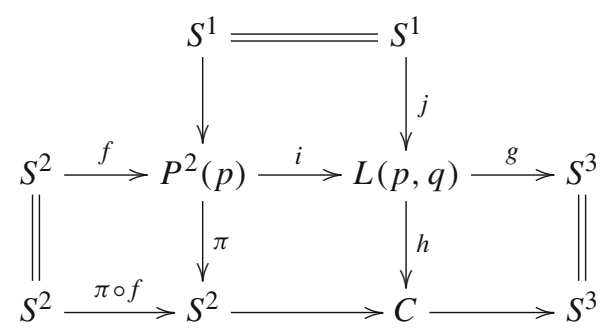


that defines the space $C$ and the maps $g$ and $h$.

Lemma 2.1 The map $\pi \circ f$ is null homotopic and there is a homotopy equivalence $C \simeq S^{2} \vee S^{3}$.

Proof The degree of $\pi \circ f$ is detected by the Bockstein in the homology of $C$, but this Bockstein is zero since the corresponding Bockstein for $L(p, q)$ is zero. Therefore, $\pi \circ f \simeq *$, implying that $C \simeq S^{2} \vee S^{3}$.

Let $\bar{\pi}$ be the composite

$$
\bar{\pi}: L(p, q) \stackrel{h}{\longrightarrow} C \stackrel{\simeq}{\longrightarrow} S^{2} \vee S^{3} \longrightarrow S^{2}
$$

where the right map collapses $S^{3}$ to a point. Lemma 2.1 immediately implies the following.

Corollary 2.2 The pinchmap $P^{2}(p) \stackrel{\pi}{\longrightarrow} S^{2}$ extends across $i$ to the map $\bar{\pi}: L(p, q) \longrightarrow$ $S^{2}$.

If $n=1$ then $B U(1)$ is the Eilenberg-Mac Lane space $K(\mathbb{Z}, 2)$ and for any $C W$-complex $X$ the set $[X, B U(1)]$ has a group structure. If $n>1$, then the standard inclusion $B U(n) \longrightarrow B U(\infty)$ has homotopy fibre $U(\infty) / U(n)$ which is $2 n$-connected. Thus, if $X$ is a $C W$-complex of dimension $\leq 2 n$ then there is an isomorphism $[X, B U(n)] \cong[X, B U(\infty)]$. In particular, as $B U(\infty)$ is an infinite loop space, $[X, B U(n)]$ has a group structure. In our case, each space in the homotopy cofibration sequences $S^{1} \longrightarrow P^{2}(p) \stackrel{\pi}{\longrightarrow} S^{2} \stackrel{p}{\longrightarrow} S^{2}$ and $S^{2} \stackrel{f}{\longrightarrow} P^{2}(p) \stackrel{i}{\longrightarrow} L(p, q) \stackrel{g}{\longrightarrow} S^{3}$ has dimension $\leq 4$ so for any $n \geq 1$ we obtain exact sequences of groups

$$
\left[S^{2}, B U(n)\right] \stackrel{\underline{p}^{*}}{\longrightarrow}\left[S^{2}, B U(n)\right] \stackrel{\pi^{*}}{\longrightarrow}\left[P^{2}(p), B U(n)\right] \longrightarrow\left[S^{1}, B U(n)\right]
$$

and

$$
\left[S^{3}, B U(n)\right] \stackrel{g^{*}}{\longrightarrow}[L(p, q), B U(n)] \stackrel{i^{*}}{\longrightarrow}\left[P^{2}(p), B U(n)\right] \stackrel{f^{*}}{\longrightarrow}\left[S^{2}, B U(n)\right] .
$$

Recall that $\left[S^{2}, B U(n)\right] \cong \pi_{1}(U(n)) \cong \mathbb{Z}$.

Lemma 2.3 Let $n \geq 1$. The following hold:

(a) there is a group isomorphism $\left[P^{2}(p), B U(n)\right] \cong \mathbb{Z} / p \mathbb{Z}$;

(b) the map $\pi^{*}$ is reduction mod-p;

(c) there is a group isomorphism $[L(p, q), B U(n)] \stackrel{i^{*}}{\longrightarrow}\left[P^{2}(p), B U(n)\right]$;

(d) the map $\bar{\pi}^{*}$ is reduction mod-p.

Proof In (2), since $p$ is the map of degree $p$, the induced map $p^{*}$ is multiplication by p. As $\pi_{2}(B U(n)) \cong \mathbb{Z}$ and $\pi_{1}(B U(n)) \cong 0$, exactness in (2) immediately implies that $\left[P^{2}(p), B U(n)\right] \cong \mathbb{Z} / p \mathbb{Z}$ and $\pi^{*}$ is reduction mod- $p$, proving parts (a) and (b). 
As $\pi_{3}(B U(n)) \cong 0,\left[P^{2}(p), B U(n)\right] \cong \mathbb{Z} / p \mathbb{Z}$ and $\left[S^{2}, B U(n)\right] \cong \mathbb{Z}$, from (3) we obtain an exact sequence of groups

$$
0 \longrightarrow[L(p, q), B U(n)] \stackrel{i^{*}}{\longrightarrow} \mathbb{Z} / p \mathbb{Z} \longrightarrow \mathbb{Z}
$$

Any homomorphism from a finite group to $\mathbb{Z}$ is trivial so, by exactness, $i^{*}$ is an isomorphism, proving part (c).

Since $\pi \simeq \bar{\pi} \circ i$ by Corollay 2.2, part (d) follows from parts (b) and (c).

In general, if $X$ is a pointed $C W$-complex then the isomorphism classes of principal $U(n)$-bundles over $X$ are classified by the homotopy classes in $[X, B U(n)]$. If $P$ is such a bundle, classified by a map $\alpha$, let $\mathcal{G}_{\alpha}(X)$ be its gauge group. This group has a classifying space $B \mathcal{G}_{\alpha}(X)$ and by [2,4] there is a homotopy equivalence $B \mathcal{G}_{\alpha}(X) \simeq \operatorname{Map}_{\alpha}(X, B U(n))$, where $\operatorname{Map}_{\alpha}(X, B U(n))$ is the component of the space of continuous maps from $X$ to $B U(n)$ that contains $\alpha$. The subgroup $\mathcal{G}_{\alpha}^{*}(X)$ of $G$-equivariant automorphisms of $P$ that pointwise fix the fibre at the basepoint is the pointed gauge group. There is a corresponding homotopy equivalence $B \mathcal{G}_{\alpha}^{*}(X) \simeq \operatorname{Map}_{\alpha}^{*}(X, B U(n))$, where $\operatorname{Map}_{\alpha}^{*}(X, B U(n))$ is the component of the continuous, pointed maps from $X$ to $B U(n)$ that contains $\alpha$. Evaluation of maps at the basepoint gives a homotopy fibration sequence

$$
U(n) \stackrel{\partial_{\alpha}}{\longrightarrow} \operatorname{Map}_{\alpha}^{*}(X, B U(n)) \longrightarrow \operatorname{Map}_{\alpha}(X, B U(n)) \longrightarrow B U(n)
$$

The homotopy fibre of the connecting map $\partial_{\alpha}$ is $\mathcal{G}_{\alpha}(X)$.

In our case, we have $\left[S^{2}, B U(n)\right] \cong \mathbb{Z}$ and, by Lemma $2.3,\left[P^{2}(p), B U(n)\right] \cong$ $[L(p, q), B U(n)] \cong \mathbb{Z} / p \mathbb{Z}$. Note that, for dimensional reasons, the principal $U(n)$ bundles over $S^{2}, P^{2}(p)$ and $L(p, q)$ are classified by the value of the first Chern class. For $\bar{k} \in \mathbb{Z}$, let $\mathcal{G}_{\bar{k}}\left(S^{2}\right)$ be the gauge group of the isomorphism class of principal $U(n)$-bundles over $S^{2}$ whose first Chern class is $\bar{k}$. For $k \in \mathbb{Z} / p \mathbb{Z}$, let $\mathcal{G}_{k}\left(P^{2}(p)\right)$ and $\mathcal{G}_{k}(L(p, q))$ be the respective gauge groups of the isomorphism classes of principal $U(n)$-bundles over $P^{2}(p)$ and $L(p, q)$ whose first Chern class is $k$. Lemma 2.3 implies that if $\bar{k} \equiv k \bmod p$ then there is a commutative diagram of fibration sequences

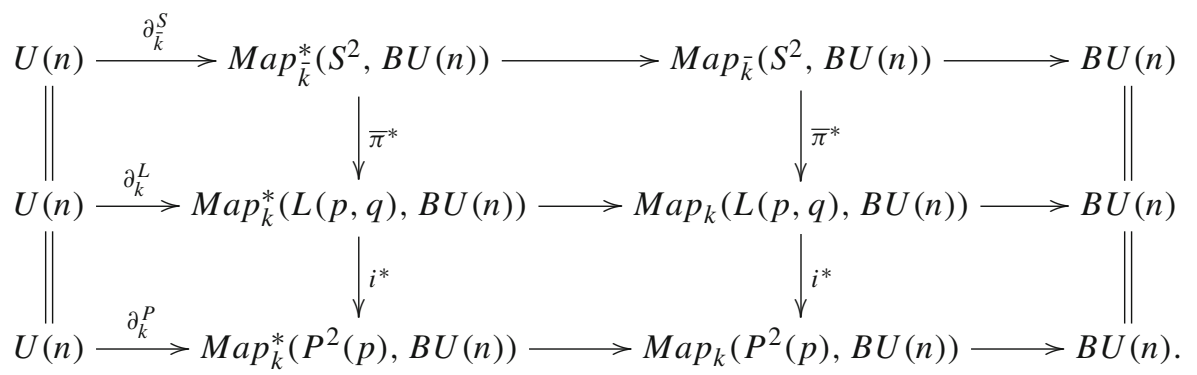

The homotopy fibres of $\partial_{\bar{k}}^{S}, \partial_{k}^{L}$ and $\partial_{k}^{P}$ are $\mathcal{G}_{\bar{k}}\left(S^{2}\right), \mathcal{G}_{k}(L(p, q))$ and $\mathcal{G}_{k}\left(P^{2}(p)\right)$ respectively. 
The goal is to find information about the gauge groups $\mathcal{G}_{k}(L(p, q))$ via the middle homotopy fibration in (4). However, it is not so easy to study this fibration directly, one issue being that it is unclear whether the components $\operatorname{Map}_{k}^{*}(L(p, q), B U(n))$ are all homotopy equivalent. A similar issue appeared in work of the first author [5] in dealing with gauge groups for principal $G$-bundles over $S^{3}$-bundles over $S^{4}$, where $G$ is a simply-connected, simple compact Lie group. The approach in that case involved localization, which needs to be avoided here since $P^{2}(p)$ need not be nilpotent. Instead, we obtain information through self-equivalences of $P^{2}(p)$ and $L(p, q)$ and how these interact with known information from Ref. [7] about $\mathcal{G}_{\bar{k}}\left(S^{2}\right)$ via the top fibration in (4).

There is another way of viewing gauge groups that will also be helpful. The following argument was suggested by a referee. The gauge group of a principal $G$-bundle $P \rightarrow M$ can be identified with the space of sections $\Gamma\left(P \times{ }_{G} G\right)$, where the action of $G$ on itself is the adjoint one. Since $P \times_{G} G \cong P / Z \times_{G / Z} G$, where $Z$ is the center of $G$, it follows that the gauge group is determined by the principle $G / Z$-bundle $P / Z \rightarrow M$. In our case, $G / Z=P U(n)$. Using exact sequences similar to (2) and (3) we get

$$
[L(p, q), B P U(n)] \cong\left[P^{2}(p), B P U(n)\right] \cong \mathbb{Z} /(n, p) \mathbb{Z}
$$

Since all $P U(n)$-bundles are obtained as adjoint bundles of $U(n)$-bundles we obtain the following.

Lemma 2.4 If $k, l \in \mathbb{Z} / p \mathbb{Z}$ are such that $k \equiv l(\bmod (p, n))$ then $\mathcal{G}_{k}\left(P^{2}(p)\right) \simeq$ $\mathcal{G}_{l}\left(P^{2}(p)\right)$ and $\mathcal{G}_{k}(L(p, q)) \simeq \mathcal{G}_{l}(L(p, q))$.

Notably, Lemma 2.4 implies that if $p$ and $n$ are coprime then $\mathcal{G}_{k}\left(P^{2}(p)\right) \simeq$ $\mathcal{G}_{0}\left(P^{2}(p)\right)$ and $\mathcal{G}_{k}(L(p, q)) \simeq \mathcal{G}_{0}(L(p, q))$.

\section{The homotopy types of $\mathcal{G}_{k}\left(P^{2}(p)\right)$}

By Corollary 2.2, the pinch map $P^{2}(p) \stackrel{\pi}{\longrightarrow} S^{2}$ is homotopic to the composite $P^{2}(p) \stackrel{i}{\longrightarrow} L(p, q) \stackrel{\bar{\pi}}{\longrightarrow} S^{2}$. Therefore, from (4), we obtain a homotopy commutative diagram of fibration sequences

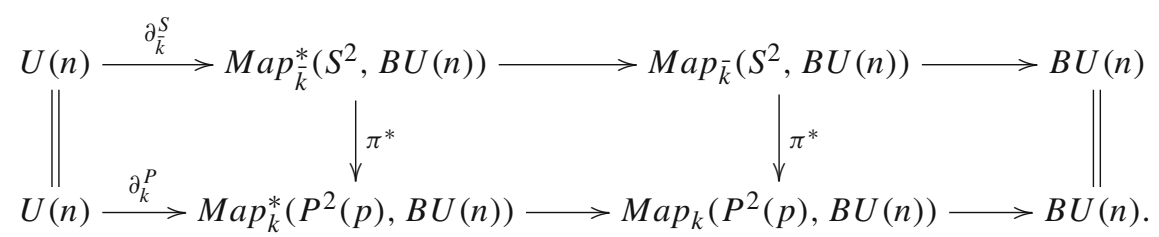

First, we show that all the components $\operatorname{Map}_{k}^{*}\left(P^{2}(p), B U(n)\right)$ are homotopy equivalent, and in a way that is compatible with a similar result from Ref. [7] about the components $\operatorname{Map}_{\bar{k}}^{*}\left(S^{2}, B U(n)\right)$. In terms of gauge groups, this says that all the classifying spaces $B \mathcal{G}_{k}^{*}\left(P^{2}(p)\right)$ of the pointed gauge groups are homotopy equivalent, and in a way compatible with the equivalences of the classifying spaces $B \mathcal{G}_{\bar{k}}^{*}\left(S^{2}\right)$. 
Lemma 3.1 For $\bar{k} \in \mathbb{Z}$ and $k \in \mathbb{Z} / p \mathbb{Z}$ with $\bar{k} \equiv k \bmod p$, there is a homotopy commutative diagram

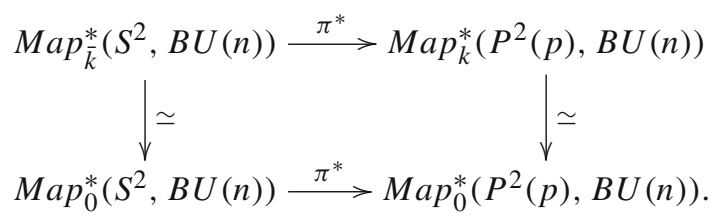

Proof This was essentially proved in Ref. [7] but not stated in this form. An argument is given for the sake of completeness. Let $\epsilon: S^{2} \longrightarrow B U(n)$ be a fixed map with first Chern class $-\bar{k}$. Define

$$
\theta: \operatorname{Map}_{\bar{k}}^{*}\left(S^{2}, B U(n)\right) \longrightarrow \operatorname{Map}_{0}^{*}\left(S^{2}, B U(n)\right)
$$

by sending a map $f: S^{2} \longrightarrow B U(n)$ with first Chern class $\bar{k}$ to the composite

$$
\theta(f): S^{2} \stackrel{\sigma}{\longrightarrow} S^{2} \vee S^{2} \stackrel{f \vee \epsilon}{\longrightarrow} B U(n) \vee B U(n) \stackrel{\nabla}{\longrightarrow} B U(n)
$$

where $\sigma$ is the comultiplication on $S^{2}$ and $\nabla$ is the folding map. Similarly, define

$$
\phi: \operatorname{Map}_{0}^{*}\left(S^{2}, B U(n)\right) \longrightarrow \operatorname{Map}_{\bar{k}}^{*}\left(S^{2}, B U(n)\right)
$$

by sending $g$ to $\nabla \circ(g \vee(-\epsilon)) \circ \sigma$. Then, $\theta$ and $\phi$ are continuous and the homotopy associativity of $\sigma$ implies that $\phi \circ \theta$ and $\theta \circ \phi$ are homotopic to the identity maps.

The space $P^{2}(p)$ is not a co-H-space. However, as $\pi$ is a homotopy cofibration connecting map, there is a coaction $\psi: P^{2}(p) \longrightarrow P^{2}(p) \vee S^{2}$ which, when pinched to $P^{2}(p)$ is the identity map, and when pinched to $S^{2}$ is $\pi$. Furthermore, this coaction has a homotopy associativity property: $(\psi \vee 1) \circ \psi \simeq 1 \vee \sigma$. Define

$$
\theta^{\prime}: \operatorname{Map}_{k}^{*}\left(P^{2}(p), B U(n)\right) \longrightarrow \operatorname{Map}_{0}^{*}\left(P^{2}(p), B U(n)\right)
$$

by sending a map $f^{\prime}: P^{2}(p) \longrightarrow B U(n)$ with first Chern class $k$ to the composite

$$
\theta^{\prime}(f): P^{2}(p) \stackrel{\psi}{\longrightarrow} P^{2}(p) \vee S^{2} \stackrel{f^{\prime} \vee \epsilon}{\longrightarrow} B U(n) \vee B U(n) \stackrel{\nabla}{\longrightarrow} B U(n)
$$

and define

$$
\phi^{\prime}: \operatorname{Map}_{0}^{*}\left(P^{2}(p), B U(n)\right) \longrightarrow \operatorname{Map}_{k}^{*}\left(P^{2}(p), B U(n)\right)
$$

by sending $g$ to $\nabla \circ(g \vee(-\epsilon)) \circ \psi$. Then, as before, $\theta^{\prime}$ is a homotopy equivalence. 
Finally, the coaction $\psi$ satisfies a homotopy commutative diagram

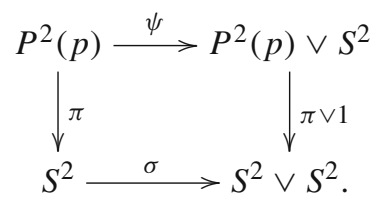

This implies that $\theta$ and $\theta^{\prime}$, and $\phi$ and $\phi^{\prime}$, are compatible, implying the homotopy commutative diagram asserted by the lemma.

Using $\partial_{\bar{k}}^{S}$ to also denote the composite $U(n) \stackrel{\partial_{\bar{k}}^{S}}{\longrightarrow} \operatorname{Map}_{\bar{k}}^{*}\left(S^{2}, B U(n)\right) \stackrel{\simeq}{\longrightarrow}$ $\operatorname{Map}_{0}^{*}\left(S^{2}, B U(n)\right)$, and similarly for $\partial_{k}^{P}$, by Lemma 3.1 the left square in (5) may be replaced with a homotopy commutative square

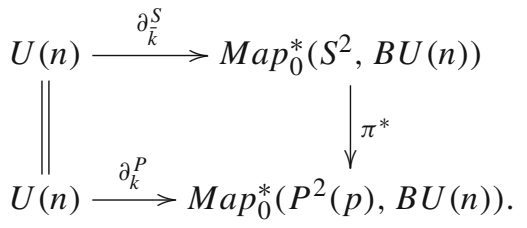

By (5), the homotopy fibres of $\partial_{\bar{k}}^{S}$ and $\partial_{k}^{P}$ are $\mathcal{G}_{\bar{k}}\left(S^{2}\right)$ and $\mathcal{G}_{k}\left(P^{2}(p)\right)$ respectively.

We next identify certain self-homotopy equivalences of $\operatorname{Map}_{k}^{*}\left(P^{2}(p), B U(n)\right)$. Since $P^{2}(p)$ is not a co- $H$-space it is not immediately clear that it has a degree $d$ map for any integer $d$. However, Olum [6] [Theorem 6.2] has shown that there are analogous maps and they behave the way one would hope.

Lemma 3.2 If $d$ is a unit mod- $p$ then there is a homotopy equivalence $\underline{d}: P^{2}(p) \longrightarrow$ $P^{2}(p)$ satisfying a homotopy commutative diagram

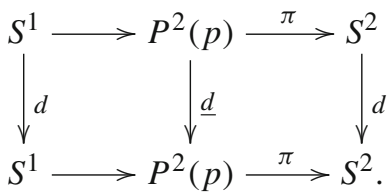

Since $\underline{d}$ is a homotopy equivalence, it induces a homotopy equivalence

$$
\operatorname{Map}_{k}\left(P^{2}(p), B U(n)\right) \longrightarrow \operatorname{Map}_{d k}\left(P^{2}(p), B U(n)\right)
$$

Phrased in terms of gauge groups this gives the following.

Corollary 3.3 If is a unit mod-p then there is a homotopy equivalence $B \mathcal{G}_{k}\left(P^{2}(p)\right) \simeq$ $B \mathcal{G}_{d k}\left(P^{2}(p)\right)$. 
Recall that, by elementary number theory, if $(u, n)=1$, then $u$ is a unit $\bmod n$, and if $(k, n)=(\ell, n)$, then $k \equiv u \ell \bmod n$ for some integer $u$ satisfying $(u, n)=1$.

Proposition 3.4 Let $r=(p, n)$. Suppose that $(k, r)=(\ell, r)$, implying that $k \equiv$ $u \ell \bmod r$ for some integer satisfying $(u, r)=1$. Suppose that $(u, p)=1$ as well. Then, $\mathcal{G}_{k}\left(P^{2}(p)\right) \simeq \mathcal{G}_{\ell}\left(P^{2}(p)\right)$.

Proof Since $r=(p, n)$, the fact that $k \equiv u \ell \bmod r$ lets us apply Lemma 2.4 to obtain a homotopy equivalence $\mathcal{G}_{k}\left(P^{2}(p)\right) \simeq \mathcal{G}_{u \ell}\left(P^{2}(p)\right)$. Since $u$ is a unit mod- $p$, by Corollary 3.3 there is a homotopy equivalence $\mathcal{G}_{\ell}\left(P^{2}(p)\right) \simeq \mathcal{G}_{u \ell}\left(P^{2}(p)\right)$. Putting these together gives $\mathcal{G}_{k}\left(P^{2}(p)\right) \simeq \mathcal{G}_{\ell}\left(P^{2}(p)\right)$.

There is a partial converse to Proposition 3.4 in a limited number of cases.

Lemma 3.5 There is an isomorphism $\pi_{2 n-1}\left(B \mathcal{G}_{k}\left(P^{2}(p)\right)\right) \cong \mathbb{Z} /(p,(n-1) !(n, k)) \mathbb{Z}$.

Proof The homotopy cofibration $P^{2}(p) \stackrel{\pi}{\longrightarrow} S^{2} \stackrel{p}{\longrightarrow} S^{2}$ induces an exact sequence

$$
\begin{aligned}
& \pi_{2 n-1}\left(\Omega_{0} U(n)\right) \stackrel{p}{\longrightarrow} \pi_{2 n-1}\left(\Omega_{0} U(n)\right) \stackrel{\pi^{*}}{\longrightarrow} \pi_{2 n-1}\left(\operatorname{Map}_{0}^{*}\left(P^{2}(p), B U(n)\right)\right) \\
& \longrightarrow \pi_{2 n-2}\left(\Omega_{0} U(n)\right) \stackrel{p}{\longrightarrow} \pi_{2 n-2}\left(\Omega_{0} U(n)\right) .
\end{aligned}
$$

By Refs. [3] or [8], $\pi_{2 n}(U(n)) \cong \mathbb{Z} / n ! \mathbb{Z}$, and it is well known that $\pi_{2 n-1}(U(n)) \cong \mathbb{Z}$. As multiplication by $p$ on $\mathbb{Z} / n ! \mathbb{Z}$ sends a generator $\gamma$ to $(n !, p) \gamma$ and mutliplication by $p$ on $\mathbb{Z}$ is an injection, we obtain $\pi_{2 n-1}\left(\operatorname{Map}_{0}^{*}\left(P^{2}(p), B U(n)\right)\right) \cong \mathbb{Z} /(n !, p) \mathbb{Z}$ and $\pi^{*}$ is reduction $\bmod (n !, p)$.

Next, consider the commutative diagram

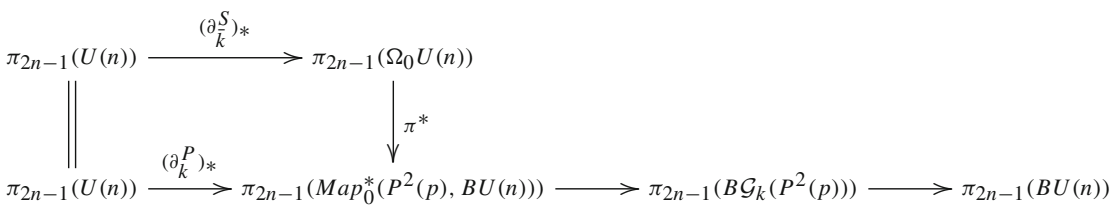

induced by (6), and note that the bottom row is exact. The fact that $\pi_{2 n-1}(B U(n)) \cong 0$ implies that $\pi_{2 n-1}\left(B \mathcal{G}_{k}\left(P^{2}(p)\right)\right)$ is isomorphic to the cokernel of $\left(\partial_{k}^{P}\right)_{*}$. We wish to identify this cokernel in a manner related to $(n, k)$ and then compare to the $(n, \ell)$ case.

Sutherland [7] showed that the image of $\left(\partial \frac{S}{k}\right)_{*}$ is generated by $(n-1) !(n, \bar{k}) \gamma$. Let $\delta=\pi^{*}(\gamma)$. Then the image of $\left(\partial_{k}^{P}\right)_{*}$ is generated by $(n-1) !(n, k) \delta$. Therefore the cokernel of $\left(\partial_{k}^{P}\right)_{*}$ is isomorphic to the cokernel of the map $\mathbb{Z} \stackrel{(n-1) !(n, k)}{\longrightarrow} \mathbb{Z} /(n !, p) \mathbb{Z}$, which is $\mathbb{Z} /((n !, p),(n-1) !(n, k)) \mathbb{Z}$. Since $((n !, p),(n-1) !(n, k))=(p,(n-$ $1) !(n, k))$ we obtain $\pi_{2 n-1}\left(B \mathcal{G}_{k}\left(P^{2}(p)\right)\right) \cong \mathbb{Z} /(p,(n-1) !(n, k)) \mathbb{Z}$.

Corollary 3.6 If $\mathcal{G}_{k}\left(P^{2}(p)\right) \simeq \mathcal{G}_{\ell}\left(P^{2}(p)\right)$ then $(p,(n-1) !(n, k))=(p,(n-$ $1) !(n, \ell))$.

Proposition 3.4 and Corollary 3.6 can be combined to give a complete classification of the homotopy types of the gauge groups $\mathcal{G}_{k}\left(P^{2}(p)\right)$ in a special case. 
Proof of Theorem 1.1 (a) Suppose that $\mathcal{G}_{k}\left(P^{2}(p)\right) \simeq \mathcal{G}_{\ell}\left(P^{2}(p)\right)$. Then, as $n=p$, by Corollary $3.6(p,(p-1) !(p, k))=(p,(p-1) !(p, \ell))$. Since $p$ is a prime, $(p-1)$ ! and $p$ are coprime so $(p,(p-1) !(p, k))=(p,(p, k))=(p, k)$. Similarly, $(p,(p-$ $1) !(p, \ell))=(p, \ell)$. Hence $(p, k)=(p, \ell)$.

Conversely, suppose that $(p, k)=(p, \ell)$. In Proposition 3.4, as $p=n$ we have $r=(p, n)=(p, p)=p$, so the two conditions $(u, r)=1$ and $(u, p)=1$ coincide. The statement of the proposition now says that if $(p, k)=(p, \ell)$ then $\mathcal{G}_{k}\left(P^{2}(p)\right) \simeq$ $\mathcal{G}_{\ell}\left(P^{2}(p)\right)$.

\section{The homotopy types of $\mathcal{G}_{k}(L(p, q))$}

The strategy and results obtained are similar to those for $\mathcal{G}_{k}\left(P^{2}(p)\right)$. This begins with identifying self-homotopy equivalences of lens spaces, which require an additional condition as compared to those for two-dimensional Moore spaces. Consider the homotopy cofibration $S^{2} \longrightarrow P^{2}(p) \longrightarrow L(p, q)$ that attaches the top cell to $L(p, q)$. The following was established in Ref. [6] [Remark 7.4].

Lemma 4.1 Suppose that $u$ is a unit mod- $p$ with the property that $u^{2} \equiv \pm 1 \bmod p$. Then, there is a homotopy cofibration diagram

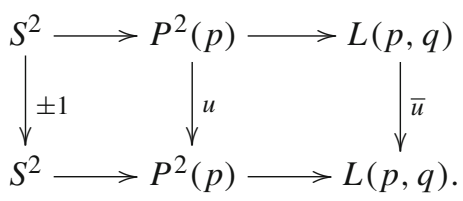

where $\bar{u}$ is a homotopy equivalence.

Since $\bar{u}$ is a homotopy equivalence, it induces a homotopy equivalence

$$
\operatorname{Map}_{k}(L(p, q), B U(n)) \longrightarrow \operatorname{Map}_{u k}(L(p, q), B U(n)) .
$$

Phrased in terms of gauge groups this gives the following.

Corollary 4.2 If $u$ is a unit mod- $p$ with the property that $u^{2} \equiv \pm 1 \bmod p$ then there is a homotopy equivalence $B \mathcal{G}_{k}(L(p, q)) \simeq B \mathcal{G}_{u k}(L(p, q))$.

Similar to Proposition 3.4, we obtain the following.

Proposition 4.3 Let $r=(p, n)$. Suppose that $(k, r)=(\ell, r)$, implying that $k \equiv$ $u \ell$ mod $r$ for some integer satisfying $(u, r)=1$. Suppose in addition that $(u, p)=1$ and $u^{2} \equiv \pm 1 \bmod p$. Then, there is a homotopy equivalence $\mathcal{G}_{k}(L(p, q)) \simeq$ $\mathcal{G}_{\ell}(L(p, q))$.

Proof Since $r=(p, n)$, the fact that $k \equiv u \ell \bmod r$ lets us apply Lemma 2.4 to obtain a homotopy equivalence $\mathcal{G}_{k}(L(p, q)) \simeq \mathcal{G}_{u \ell}(L(p, q))$. Since $u$ is a unit mod- $p$ and $u^{2} \equiv \pm 1 \bmod p$, by Corollary 4.2 there is a homotopy equivalence $\mathcal{G}_{\ell}(L(p, q)) \simeq$ $\mathcal{G}_{u \ell}(L(p, q))$. Putting these together gives $\mathcal{G}_{k}(L(p, q)) \simeq \mathcal{G}_{\ell}(L(p, q))$. 
Conversely, starting with a homotopy equivalence $\mathcal{G}_{k}(L(p, q)) \simeq \mathcal{G}_{\ell}(L(p, q))$ we aim for a g.c.d. condition involving $k, \ell, n$ and $p$. Consider the homotopy cofibration sequence

$$
S^{2} \stackrel{f}{\longrightarrow} P^{2}(p) \stackrel{i}{\longrightarrow} L(p, q) \stackrel{g}{\longrightarrow} S^{3} .
$$

Lemma 4.4 There is a homotopy equivalence $\Sigma^{2} L(p, q) \simeq P^{4}(p) \vee S^{5}$.

Proof In general, any closed, orientable 3-manifold $M$ is parallelizable so by Ref. [1] it has the property that its top cell splits off stably. In our case, as $L(p, q)$ is such a manifold, the attaching map $f$ for the top cell is stably trivial. As $f$ is in the stable range after two suspensions, this implies that $\Sigma^{2} f$ null homotopic. Thus, there is a homotopy equivalence $\Sigma^{2} L(p, q) \simeq P^{4}(p) \vee S^{5}$.

Lemma 4.5 If $n>1$ there is an isomorphism

$$
\pi_{2 n-1}\left(B \mathcal{G}_{k}(L(p, q))\right) \cong \begin{cases}\mathbb{Z} /(p,(n-1) !(n, k)) \mathbb{Z} & \text { ifnis odd } \\ \mathbb{Z} /(p,(n-1) !(n, k)) \mathbb{Z} \oplus \mathbb{Z} / 2 \mathbb{Z} \text { ifnis even } .\end{cases}
$$

Proof It is worth first pointing out that this argument is different from that for Lemma 3.5, since it is not clear that the components $\operatorname{Map}_{k}^{*}(L(p, q), B U(n))$ are all homotopy equivalent, so a different approach is needed. Consider the homotopy cofibration diagram

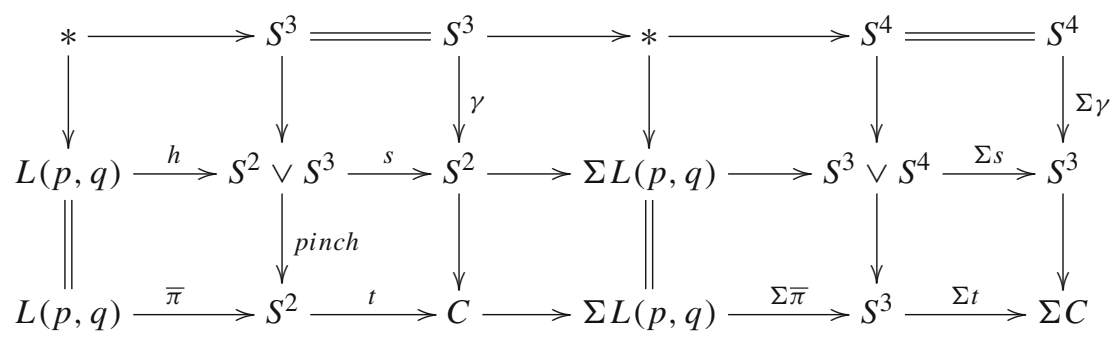

which defines the space $C$ and the maps $s, t$ and $\gamma$. The restriction of $s$ to $S^{2}$ is the degree $p$ map and its restriction to $S^{3}$ is $a \cdot \eta$, where $\eta$ represents a generator of $\pi_{3}\left(S^{2}\right) \cong \mathbb{Z}$. Thus $\gamma=a \cdot \eta$. Since the Steenrod square $S q^{2}$ detects $\eta$, if $a$ was odd then there would exist a nontrivial $S q^{2}$ in the mod-2 cohomology of $\Sigma L(p, q)$. This operation is stable, so would also appear in the mod-2 cohomology of $\Sigma^{2} L(p, q)$, contradicting the homotopy equivalence in Lemma 4.4. Therefore, $a$ is even. Since $\Sigma \eta$ has order 2, this implies that $\Sigma \gamma=a \cdot \Sigma \eta$ is null homotopic. Hence, $\Sigma C \simeq S^{3} \vee S^{5}$ and $\Sigma t$ is homotopic to the composite $S^{3} \stackrel{p}{\longrightarrow} S^{3} \hookrightarrow S^{3} \vee S^{5}$.

Now arguing along the lines of Ref.[5] [Lemma 4.7] (but integrally instead of $p$-locally), there is a homotopy fibration

$$
\Omega^{5} B U(n) \times \Omega^{3} B U(n) \stackrel{* \times p}{\longrightarrow} \Omega^{3} B U(n) \stackrel{\widehat{\pi}_{k}^{*}}{\longrightarrow} \Omega_{\operatorname{Map}_{k}^{*}}^{*}(L(p, q), B U(n)),
$$


where $p$ is the $p$-th power map, and the map $\widehat{\pi}_{k}^{*}$ is identified with the composite

$$
\operatorname{SMap}_{0}^{*}\left(S^{2}, B U(n)\right) \stackrel{\simeq}{\rightarrow} \Omega_{\operatorname{Map}_{k}^{*}}\left(S^{2}, B U(n)\right) \stackrel{\Omega \bar{\pi}^{*}}{\rightarrow} \Omega \operatorname{Map}_{k}^{*}(L(p, q), B U(n)) .
$$

On one hand, since $\operatorname{Map}_{k}^{*}(L(p, q), B U(n)) \simeq B \mathcal{G}_{k}(L(p, q))$, the homotopy fibration (8) implies that the homotopy fibre of $* \times p$ is $\Omega \mathcal{G}_{k}^{*}(L(p, q))$. On the other hand, working directly from the map $* \times p$, its homotopy fibre is $\Omega^{2} \operatorname{Map}_{k}^{*}\left(P^{2}(p), B U(n)\right) \times$ $\Omega^{4} U(n)$. Thus, $\Omega_{\mathcal{G}_{k}^{*}}(L(p, q)) \simeq \Omega^{2} \operatorname{Map}_{k}^{*}\left(P^{2}(p), B U(n)\right) \times \Omega^{4} U(n)$. Since $\operatorname{Map}_{k}^{*}\left(P^{2}(p), B U(n)\right) \simeq B \mathcal{G}_{k}\left(P^{2}(p)\right)$, we obtain an isomorphism

$$
\pi_{m}\left(B \mathcal{G}_{k}(L(p, q))\right) \cong \pi_{m}\left(B \mathcal{G}_{k}\left(P^{2}(p)\right)\right) \oplus \pi_{m}\left(\Omega^{2} U(n)\right)
$$

for every $m \geq 2$. In particular, if $m=2 n-1$ then $\pi_{m}\left(B \mathcal{G}_{k}\left(P^{2}(p)\right)\right) \cong \mathbb{Z} /(p,(n-$ $1) !(n, k)) \mathbb{Z}$ by Lemma 3.5 , and $\pi_{2 n-1}\left(\Omega^{2} U(n)\right) \cong 0$ if $n$ is odd and $\pi_{2 n-1}\left(\Omega^{2} U(n)\right) \cong$ $\mathbb{Z} / 2 \mathbb{Z}$ if $n$ is even by Ref. [8]. The asserted isomorphism follows.

Corollary 4.6 If $\mathcal{G}_{k}(L(p, q)) \simeq \mathcal{G}_{\ell}(L(p, q))$ then $(p,(n-1) !(n, k))=(p,(n-$ $1) !(n, \ell))$.

Proposition 4.3 and Corollary 4.6 can be combined in a special case to partially classify the homotopy types of the gauge groups $\mathcal{G}_{k}(L(p, q))$.

Proposition 4.7 Let $p$ be a prime and consider the gauge groups of principal $U(p)$ bundles over $L(p, q)$. The following hold:

(a) if $\mathcal{G}_{k}(L(p, q)) \simeq \mathcal{G}_{\ell}(L(p, q))$ then $(p, k)=(p, \ell)$;

(b) if $(p, k)=(p, \ell)$, so that $k \equiv u \ell \bmod p$ for some integer $u$ satisfying $(u, p)=1$, and if $u^{2} \equiv \pm 1 \bmod p$, then $\mathcal{G}_{k}(L(p, q)) \simeq \mathcal{G}_{\ell}(L(p, q))$.

Proof Suppose that $\mathcal{G}_{k}(L(p, q)) \simeq \mathcal{G}_{\ell}(L(p, q))$. If $p=2$ then, with $n=2$ as well, the number $(p,(n-1) !(n, k))$ in Corollary 4.6 becomes $(2,(2, k))=(2, k)$. Therefore, Corollary 4.6 implies that there is an isomorphism $\mathbb{Z} /(2, k) \mathbb{Z} \oplus \mathbb{Z} / 2 \mathbb{Z} \cong$ $\mathbb{Z} /(2, \ell) \mathbb{Z} \oplus \mathbb{Z} / 2 \mathbb{Z}$. This is only possible if $(2, k)=(2, \ell)$. If $p$ is odd, then with $n=p$, the number $(p,(p-1) !(p, k))$ in Corollary 4.6 equals $(p,(p, k))=(p, k)$ since $p$ and $(p-1)$ ! are coprime. Therefore, Corollary 4.6 implies that $(p, k)=(p, \ell)$.

Conversely, suppose that $(p, k)=(p, \ell)$. In Proposition 4.3, as $p=n$, we obtain $r=(p, n)=(p, p)=p$, so the two conditions $(u, r)=1$ and $(u, p)=1$ coincide. The hypothesis that $u^{2} \equiv \pm 1 \bmod p$ then allows Proposition 4.3 to apply to obtain $\mathcal{G}_{k}(L(p, q)) \simeq \mathcal{G}_{\ell}(L(p, q))$.

In special cases, there is a complete classification of the gauge groups of lens spaces.

Proof of Theorem 1.1 (b) If $p=3$ or $p=5$ then every unit in $\mathbb{Z} / p \mathbb{Z}$ has the property that it squares to $\pm 1 \bmod p$. Proposition 4.7 therefore implies that $\mathcal{G}_{k}(L(p, q)) \simeq$ $\mathcal{G}_{\ell}(L(p, q))$ if and only if $(p, k)=(p, \ell)$.

Acknowledgements The first author was supported by the Grace Chisholm Young Fellowship of the London Mathematical Society. 
Open Access This article is licensed under a Creative Commons Attribution 4.0 International License, which permits use, sharing, adaptation, distribution and reproduction in any medium or format, as long as you give appropriate credit to the original author(s) and the source, provide a link to the Creative Commons licence, and indicate if changes were made. The images or other third party material in this article are included in the article's Creative Commons licence, unless indicated otherwise in a credit line to the material. If material is not included in the article's Creative Commons licence and your intended use is not permitted by statutory regulation or exceeds the permitted use, you will need to obtain permission directly from the copyright holder. To view a copy of this licence, visit http://creativecommons.org/licenses/by/4.0/.

\section{References}

1. Atiyah, M.F.: Thom complexes. Proc. Lond. Math. Soc. 11, 291-310 (1961)

2. Atiyah, M.F., Bott, R.: The Yang-Mills equations over Riemann surfaces. Philos. Trans. Roy. Soc. Lond. Ser. A 308, 523-615 (1983)

3. Borel, A., Hirzebruch, F.: Characteristic classes and homogeneous spaces: I,II. Am. Math. J. 80, 458-538 (1958); 81, 315-382 (1959)

4. Gottlieb, D.H.: Applications of bundle map theory. Trans. Am. Math. Soc. 171, 23-50 (1972)

5. Membrillo Solis, I.: Homotopy types of gauge groups related to $S^{3}$-bundles over $S^{4}$. Topol. Appl. 255, 56-85 (2019)

6. Olum, P.: Self-equivalences of pseudo-projective planes. Topology 4, 109-127 (1965)

7. Sutherland, W.A.: Function spaces related to gauge groups. Proc. Roy. Soc. Edin. 121, 185-190 (1992); 22, 1711-1719 (2011)

8. Toda, H.: A topological proof of theorems of Bott and Hirzebruch for homotopy groups of unitary groups. Mem. Coll. Sci. Univ. Kyoto Ser. A Math. 32, 103-119 (1959)

Publisher's Note Springer Nature remains neutral with regard to jurisdictional claims in published maps and institutional affiliations. 